\title{
Cardioplegia heat exchangers thermal properties
}

\author{
Erik Bartuli ${ }^{1, *}$, Ondřej Borkovec ${ }^{2}$ \\ 1 Faculty of Mechanical Engineering, Heat Transfer and Fluid Flow Laboratory, Brno University of Technology, Brno, Czech Republic, Technická \\ $2896 / 2,61669$ \\ 2 Faculty of Mechanical Engineering, Heat Transfer and Fluid Flow Laboratory, Brno University of Technology, Brno, Czech Republic, Technická \\ $2896 / 2,61669$
}

\begin{abstract}
Cardiac surgeries that involve cardiopulmonary bypass technique require a stable temperature maintenance for oxygenated blood or the cardioplegia solution. For this purpose, the heat exchangers are used. Nowadays, these exchangers consist of stainless steel tubes or bellows in a plastic shell. The blood is then warmed or cooled by water flowing in the shell around these structures. In the paper, the thermal specifications of two commercially used cardioplegia heat exchangers Capiox Cardioplegia and MYOtherm XP were evaluated and compared. For this purpose, a water was used as a substitution for blood. Both components were tested for varying flow rates in the pipes in the range 20-150 I/h and fixed flow rate $700 \mathrm{l} / \mathrm{h}$ in the shell. The thermal performances of both devices resulted similarly (up to $2.4 \mathrm{~kW}$ ) at maximal flow rate. The performance factors for low flow rates approached values close to 0.98 and with increasing flow rate decreased down to 0.50. MYOtherm XP shows lower pressure drop than Capiox Cardioplegia due to more optimal construction. The major difference between the heat exchangers was observed in overall heat transfer coefficients at maximal flow rate and resulted in $2191.3 \mathrm{~W} / \mathrm{m}^{2} \mathrm{~K}$ or $1760.2 \mathrm{~W} / \mathrm{m}^{2} \mathrm{~K}$ for MYOtherm XP or Capiox Cardioplegia, respectively.
\end{abstract}

Keywords: cardioplegia heat exchangers; thermal performance; performance factor; heat transfer coefficient; pressure drop

\section{Introduction}

Complex cardiac surgeries require the patient to be connected to the cardiopulmonary bypass machine (CPB). This technique provides the patient with oxygenated blood flow to his body while giving the surgeon a motionless, bloodless surgical field. The CPB circuit consists of arterial and venous cannulae, venous reservoir, blood oxygenator, heat exchanger (HE), pumps, arterial filter and cardioplegia delivery system [1].

It has been discovered that employing the combination of CPB and hypothermia can greatly extend the time under which it is safe to perform the surgery [2]. It reduces the oxygen required by the tissues and also increases myocardial protection and optimizes the operating conditions for the patient. For this purpose, the CPB system incorporates heat exchangers to maintain low blood temperature. Even for normothermic perfusion the heat is lost to the environment and must be compensated [3]. Nowadays, the heat exchangers are usually part of the oxygenators or can stand alone and serve as a cooling or warming unit for the mixture of cardioplegia solution and blood.

The heat exchangers are composed of two pathways, one for the perfusate (blood or cardioplegia mixture) and one for water. These pathways are separated by material that allows efficient thermal exchange between the fluids. For currently commercially used heat exchangers, stainless steel is the most popular because of its combination of good heat exchange coefficient, ease of fabrication in either pleated or tubular form, 
and biocompatibility. To control the temperature of the perfusate, water of desired temperature is circulated through the heat exchanger, cooling or warming the perfusate. To increase the efficiency, the water flows in the opposite direction relative to the perfusate, maximizing the temperature differential throughout the transit [4]. More on the design of blood heat exchangers was discussed in [5].

The temperature levels depend on the physiology of the patient. For more complex surgeries that require complete cardiac arrest and therefore deep hypothermia, the temperature can be maintained in the range down to $18-22^{\circ} \mathrm{C}$ [1], otherwise the moderate hypothermia $\left(30{ }^{\circ} \mathrm{C}\right)$ is widely used [4]. In general, the oxygen consumption has been shown to fall by about $50 \%$ for each $10^{\circ} \mathrm{C}$ drop in temperature [6].

The major property for comparison of commercially used heat exchangers are their thermal specifications e.g. thermal performance or heat transfer coefficient. Although the manufacturers provide some basic information on the conditions under which the HEs should be operated, the thermal specifications are rarely listed. The studies on this topic has been given in [3], [7], [8] or [9] but the HEs evaluated in these studies are outdated and no longer used.

In this paper two currently commercially used cardioplegia heat exchangers are evaluated in laboratory. The aim of this work is to evaluate the heat exchangers from the viewpoint of thermal performance, performance factor, pressure drop and overall heat transfer coefficient (HTC).

\section{Materials and methods}

Two commercially used blood heat exchangers (Fig. 1): Capiox Cardioplegia and MYOtherm XP were tested.
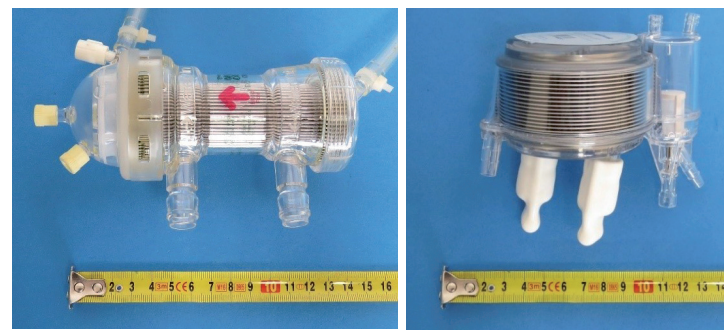

Figure 1: Capiox Cardioplegia (left) and MYOtherm XP (right).

Capiox Cardioplegia is a shell-and-tube type of
HE. Inside the polycarbonate shell there is a bundle of straight tubes made of stainless steel. The water of desired temperature flows through the shell around the tubes and warms up or cools down the blood flowing inside the tubes in the opposite direction. The length of the tube is $110 \mathrm{~mm}$ and the inner diameter is $0.9 \mathrm{~mm}$. The tubes are potted together forming a core of the HE. The diameter of the core is $55 \mathrm{~mm}$. The effective surface area of HE stated by the manufacturer is $640 \cdot 10^{-4} \mathrm{~m}^{2}$. The total volume of the device measured in laboratory is $261.3 \cdot 10^{-6} \mathrm{~m}^{3}$. The maximum blood flow rate for clinical use suggested by the manufacturer is $30 \mathrm{l} / \mathrm{h}$.

MYOtherm XP has its shell made of polycarbonate. The HE differs by its arrangement. Unlike from the previous $H E$, the heat exchange between the water and blood is carried out through a ring-shaped bellows made of stainless steel. The cooling water flows on the inner side and the blood cross-flows on the outer side of the heat exchanger. The outer diameter of the bellows is $75 \mathrm{~mm}$, the inner diameter is $64 \mathrm{~mm}$ and the height is $50 \mathrm{~mm}$. The effective surface area of HE stated by manufacturer is $600 \cdot 10^{-4} \mathrm{~m}^{2}$. The total volume of the HE measured in laboratory is $220.9 \cdot 10^{-6} \mathrm{~m}^{3}$. The maximum blood flow rate for clinical use given by the manufacturer is $30 \mathrm{l} / \mathrm{h}$.

Since blood was not available for experiments, water was chosen as the working fluid of heat exchangers. Blood and waterhave different properties, but the purpose of this article is to compare the thermal performance of heat exchangers. For this purpose, it is sufficient to use the same working fluid for both heat exchangers. The scheme of the testing device is in Fig. 2. The red line represents the circuit with warm water flowing through the heat exchanger. The water was heated to $35^{\circ} \mathrm{C}$ and pumped through the HE. The temperature was measured at the inlet and the outlet at several of flow rates in range 20-150 I/h. The pressure drop in the HE was measured by differential pressure transducer. The blue line represents the cooling water flowing through the shell. It was taken from the water system and had a temperature around $13^{\circ} \mathrm{C}$. The actual temperature was measured at the inlet and the outlet of the shell at fixed flow rate $700 \mathrm{l} / \mathrm{h}$.

The temperature probe used was RTD of class $1 / 3$ DIN. The accuracy of differential pressure transducer was $\pm 0,1 \%$ and the accuracy of flow meter was $\pm 0.25 \%$ of measured value. 


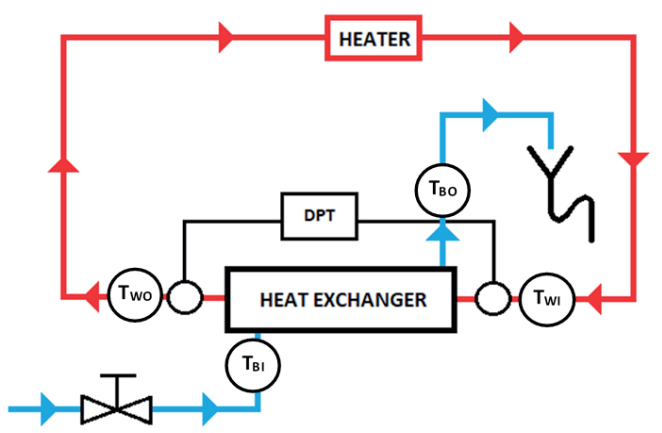

Figure 2: Scheme of the testing device (red represents water in the tubes and blue represents cooling water in the shell, $T$ is for temperature probe, DPT is for differential pressure transducer).

The thermal performance $Q$ of the heat exchangers was evaluated using a formula

$$
Q=\dot{m} c \Delta T \text {, }
$$

where $\dot{m}$ is the mass flow of the water in the heat exchanger, $c$ is the specific heat capacity of the water and $\Delta T$ is the difference of temperatures at the inlet and outlet of the HE [10].

The overall HTC $h$ was computed using formula

$$
h=\frac{Q}{S\left([T]-T_{W I}\right)} \text {, }
$$

where $\mathrm{S}$ is the effective surface area of $\mathrm{HE},[\mathrm{T}]$ is the mean of the water temperatures on the inlet and outlet of the $\mathrm{HE}$ and $\mathrm{T}_{-w 1}$ is temperature of the water at the inlet [10].

The efficiency of the heat exchangers was measured by the ratio of change in the temperature of water in the tubes to the difference between inlet temperatures of the water in the shell and water in the tubes, given by the performance factor $R$

$$
R=\frac{T_{B O}-T_{B I}}{T_{W I}-T_{B I}},
$$

where $T_{B O}$ is the temperature at the tube outlet, $T_{B 1}$ is the temperature at the tube inlet and $T_{w 1}$ is the temperature of the water at the shell inlet. The values of $R$ are in range $0-1$, where 1 corresponds to ideal heat exchanger [4], [7].

\section{Results and Discussion}

The results are shown in following graphs. The dependency of thermal performance and performance factor on the flow rate is showed in Fig. 3. The thermal performance for low flow rates resulted almost the same for both heat exchangers. The difference between heat exchangers was slightly increasing with increasing flow rate. MYOtherm XP achieved thermal performance $2.4 \mathrm{~kW}$ and Capiox achieved $2.3 \mathrm{~kW}$ for the flow rate $150 \mathrm{l} / \mathrm{h}$. The performance factor of both heat exchangers was 0.97 for flow rate $20 \mathrm{l} / \mathrm{h}$. This value further decreased with increasing flow rates down to 0.50 .

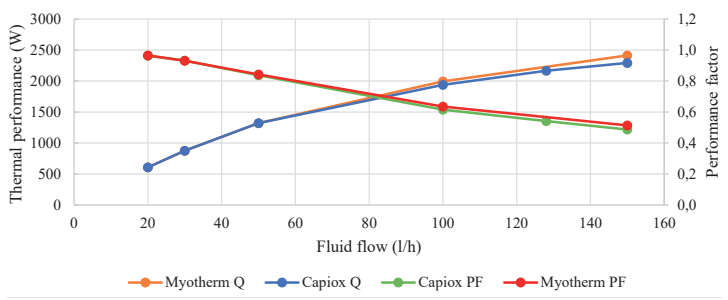

Figure 3: Dependence of thermal performance (Q) and performance factor (PF) on the flow rate.

More significant difference between heat exchangers occurred in pressure drop (Fig. 4). MYOtherm XP achieved better results due to its different construction from Capiox Cardioplegia. In comparison to tubes, the core formed by bellows shows lower resistance to the water flowing through the core of the heat exchanger. The maximum pressure drop of MYOtherm XP and Capiox Cardioplegia was $35.1 \mathrm{kPa}$ and $54.3 \mathrm{kPa}$, respectively. However, in clinical conditions, where the flow rate does not exceed $30 \mathrm{l} / \mathrm{h}$, the difference was insignificant.

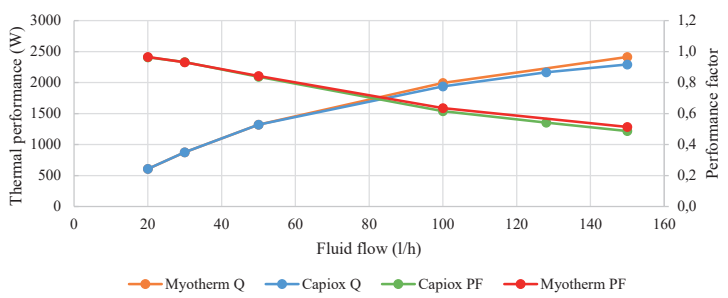

Figure 4: Dependence of the pressure drop on the flow rate.

In Fig. 5 there is a comparison of overall HTC. Depending on the flow rate, the overall HTC for Capiox Cardioplegia was about 10-19 \% smaller than for MYOtherm XP, e.g. for the flow rate 20 $\mathrm{l} / \mathrm{h}$ the achieved values were $671.2 \mathrm{~W} / \mathrm{m}^{2} \mathrm{~K}$ and $755.7 \mathrm{~W} / \mathrm{m}^{2} \mathrm{~K}$, respectively. The difference became more significant for higher flow rates. For the flow 
rates exceeding $150 \mathrm{I} / \mathrm{h}$ the values of HTC settled down to $1760.2 \mathrm{~W} / \mathrm{m}^{2} \mathrm{~K}$ for Capiox Cardioplegia and 2191.3 W/m²K for MYOtherm XP.

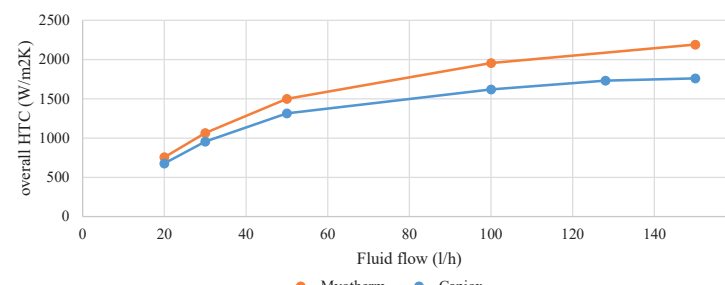

Figure 5: Profile Dependence of the overall HTC on the flow rate.

\section{Conclusions}

Two heat exchangers commercially used for cardiac surgeries were tested and compared. The heat exchangers differed in the inner structure. Capiox Cardioplegia has its core formed by tubes of diameter $0.9 \mathrm{~mm}$. MYOtherm XP has its core formed by bellows. The water of temperature $35^{\circ} \mathrm{C}$ has been used as a substitution for the blood in the heat exchanger. Each HE was evaluated for various flow rates in the tubes in a range 20-150 l/h. The water flow rate in the shell was fixed at a flow rate $700 \mathrm{l} / \mathrm{h}$. The thermal performance, overall heat transfer coefficient, pressure drop and performance factor were evaluated based on the measurements.

For low flow rates the difference between HEs was negligible. MYOtherm XP started achieving better results over Capiox Cardioplegia with an increasing flow rate. That was caused by the difference in the construction of the heat exchangers. The bellows type structure shows better results over the steel tubes structure. The maximal achieved thermal performance of the heat exchangers was $2.4 \mathrm{~kW}$ for MYOtherm XP and $2.3 \mathrm{~kW}$ for Capiox Cardioplegia. The performance factor for both heat exchangers attained the highest values close to 1 for low flow rates and with increasing flow rates decreased down to 0.5 . The most significant difference between the two heat exchangers was in overall heat transfer coefficient and pressure drop. Depending on the flow rate, the overall HTC for Capiox Cardioplegia resulted about 10-19\% smaller than for MYOtherm XP. The maximum value of HTC was $2191.3 \mathrm{~W} / \mathrm{m}^{2} \mathrm{~K}$ and $1760.2 \mathrm{~W} / \mathrm{m}^{2} \mathrm{~K}$ for MYOtherm $\mathrm{XP}$ and Capiox Cardioplegia, respectively. On top of the better results MYOtherm XP has also lower effective volume, which makes it more efficient in terms of size and material consumption during its production.

\section{Acknowledgments}

This work was supported by the Ministry of Education, Youth and Sports of the Czech Republic under OP RDE grant number CZ.02. 1.01/0.0/0.0/16_019/0000753 "Research centre for low-carbon energy technologies".

\section{References}

[1] Kiziltug, H., Martinez, G.: Cardiopulmonary bypass, Anaesth. Intensive Care Med., vol. 19, no. 7, pp. 353-360, 2018.

[2] Sealy, W., Brown, I., Young W.: A Report on the Use of Both Extracorporeal Circulation and Hypothermia for Open Heart Surgery, Ann. Surg., vol. 147, no. 5, pp. 603-613, May 1958.

[3] Bethune, D., Gill, R., Wheeldon, D.: Performance of heat exchangers used in whole body perfusion circuits, Thorax, vol. 30, no. 5, pp. 569-573, 1975.

[4] Kay, P., Munsch, C.: Techniques in Extracorporeal Circulation 4E. CRC Press, 2004.

[5] Ahuja, A., Hendee, W.: Thermal design of a heat exchanger for heating or cooling blood, Phys. Med. Biol., vol. 23, no. 5, p. 010, Sep. 1978.

[6] Kirklin, J., Barratt-Boyes, B.: Hypothermia, circulatory arrest, and cardiopulmonary bypass, in Cardiac Surgery, 1986, pp. 30-74.

[7] Drummond, M., Parorali Novello, W., Fonseca De Arruda, A., Braile, D.: Heat exchangers for cardioplegia systems: In vitro study of four different concepts, Artif. Organs, vol. 27, no. 5, pp. 465-468, 2003.

[8] Patel, N., Smith, C., Pinchak, A., Comparison of fluid warmer performance during simulated clinical conditions, Can. J. Anaesth., vol. 42, no. 7, pp. 636-642, 1995.

[9] Segers, P., Heida, J., de Vries, I., Maas, C., Boogaart, A., Eilander, S.: Clinical evaluation of nine hollow-fibre membrane oxygenators, Perfusion, vol. 16, no. 2, pp. 95-106, 2001.

[10] Incropera, F., DeWitt, D., Bergman, T., Lavine, A. Fundamentals of Heat and Mass Transfer (6th edition). 2007. 